\title{
Central Nervous System Involvement as Relapse in Undiagnosed Whipple's Disease with Atypical Symptoms at Onset
}

\author{
Ioannis Zalonis, Foteini Christidi ${ }^{*}$, Constantin Potagas, Michalis Rentzos, Ioannis Evdokimidis and \\ Evangelia Kararizou
}

Department of Neurology, Aeginition Hospital, Medical School, Athens National \& Kapodistrian University, 72-74 Vas. Sofias Avenue, Athens, 115 28, Greece

\begin{abstract}
Whipple's disease (WD) is a rare systemic disease caused by the gram-positive bacillus Tropheryma Whipplei and mostly characterized by arthralgias, chronic diarrhea, weight loss, fever and abdominal pain. Central Nervous System involvement is not uncommon and it may precede other disease manifestations, appear after treatment and improvement of gastrointestinal signs or rarely be the only WD symptom. We report a case in a middle-aged male with unexplained neurological signs and symptoms which were presented as relapse of previously undiagnosed WD with atypical symptoms at onset. After diagnosis confirmation, the patient was appropriately treated which resulted in improvement of major symptoms.
\end{abstract}

Keywords: Tropheryma whipplei, whipple's disease, central nervous system, relapse.

\section{DEAR EDITOR}

Whipple's disease (WD) is a rare systemic disease caused by the gram-positive bacillus Tropheryma Whipplei and mostly characterized by arthralgias, chronic diarrhea, weight loss, fever and abdominal pain [1]. The diagnosis depends on the detection of periodic acid-Schiff (PAS)positive macrophages and the identification of rod-shaped bacilli within these cells on electron microscopy. Polymerase chain reaction (PCR) analysis for Tropheryma Whipplei has been considered to be a non-invasive, valid diagnostic method in WD, specifically in cases of Central Nervous System (CNS) WD [2]. CNS involvement is not uncommon and it may precede other disease manifestations, appear after treatment and improvement of gastrointestinal signs or more rarely be the only WD symptom [3, 4]. Treatment approaches include antibiotics; not only they have decreased the number of deaths and morbidity but also they have prevented irreversible multisystem damage. We report a case with unexplained neurological signs and symptoms, then diagnosed with WD and appropriately treated, resulting in improvement of major symptoms.

A middle-aged Caucasian male (48 years old) was admitted to our hospital due to unexplained neurological symptoms. He had a history of a short-lasting arthritis of left ankle, unknown etiology recurring episodes of fever and one episode of persistent high fever and lymphadenopathy for which he was referred to an outpatient clinic; at that time,

*Address correspondence to this author at the Department of Neurology, Aeginition Hospital, Medical School, Athens National \& Kapodistrian University, $72-74$ Vas. Sofias Avenue, Athens, 115 28, Greece;

Tel: 0030-210-7289141; Fax: 0030-210-7250410;

E-mail: christidi.f.a@gmail.com laboratory examinations were negative with the exception of raised $\mathrm{C}$ reactive protein (CRP) and slight elevation of $\mathrm{a}_{1}$ and $\mathrm{a}_{2}$ globulins. He was given a short-duration antibiotic treatment without further episodes. During the last year he progressively presented anorexia and weight loss, myoclonic-like movements of right upper and lower extremes, gait, occulomotor and sleep disturbances, depressive mood, withdrawal, and cognitive impairment. At admission, our patient suffered from insomnia and neurological examination revealed mild ataxic walking, impaired vertical gaze, dysarthria and myoclonic type movements with right predominance. Deep tendon reflexes, pain, touch and proprioception were examined and were normal. Biochemical and immunological findings were negative. Additionally, T3, T4, TSH, thyreotropin antibody, anti-TPO, vitamin B12, Fe, AFP, CA19-9, PSA, $\beta 2$ microspherin, VDRL, FTA, VIDAL, WRIGHT were negative. Thyroid U/S was also negative. Detailed microbiological examination revealed no evidence for virus infection in blood and cerebrospinal fluid (CSF). Other diseases, like sarcoidosis, neurosyphilis and different types of hepatitis, were also excluded. There was no evidence of thrombophilia. CSF examination revealed pleocytosis 35 ( $98 \%$ lymphocytosis, $2 \%$ polynuclear), protein 51 , glucose 55. PCR for TBC mycobacter and Borrelia Burgdorferi were negative. Thoracic examination, heart and carotid triplex were also negative. EEG and brain CT findings were negative. Brain MRI (T2, FLAIR) showed three non-specific hyperintense white matter (WM) lesions in left frontal lobe and one hyperintense WM lesion in right frontal lobe, without gadolinum enhancement. Increased pathological signal intensity was found on the left parahippocampal gyrus and the subiculum $(5 \mathrm{~mm})$, with mild gadolinium enhancement. Detailed neuropsychological examination 
showed significant cognitive deterioration, with predominant memory, visuomotor and executive impairment. A duodenal biopsy specimen was not remarkable on light microscopy (PAS was negative and thus electron microscopy was not performed). Molecular detection of the 16SrRNA genes by PCR analysis of CSF, stools and blood was performed which confirmed the diagnosis. PCR analysis for Tropheryma Whipplei was positive in stools and serum. The diagnosis of WD with CNS involvement was made and our patient was started on antibiotic treatment (IV ceftriaxone for 20 days followed by oral trimethoprim/sulfamethoxazole for one year) resulted in slight improvement of major symptoms after two weeks (occulomotor function, myoclonus) and further improvement over the following six months with patient getting back to his everyday activities but without being able to resume his professional status. Brain MRI scan showed resolution of the previous mentioned lesions in the left parahippocampal gyrus, with frontal WM lesions remaining but being non-specific.

WD is a rare systemic infection that may virtually affect any organ system in the body with traditional symptoms in the small bowel. CNS involvement occurs in approximately $50 \%$ of WD postmortem examinations; CNS clinical manifestations are observed in only $20-40 \%$ of patients [5-7] whereas approximately $5 \%$ of patients present with CNS symptoms alone [5]. Relapses after treatment are not rare and CNS manifestations carry the highest relapse rate [7]. Isolated CNS manifestations presenting as relapse of previously treated WD but without histological evidence of intestinal involvement have been observed during or years after antibiotic treatment, specifically when the initial therapy is of short duration, making the treatment of relapse more difficult than the first manifestations $[8,9]$.

Our patient referred to our hospital with predominant neurological symptoms, in accordance with the WD spectrum of CNS manifestations [5, 7, 10]. At admission, he had no active gastrointestinal tract symptoms but his history included single or recurrent episodes of arthralgia, fever and lymphadenopathy. During previous admission to outpatient clinic, short-duration antibiotic treatment might result in symptoms to be relief. However, our patient was not diagnosed with WD at that time. Multiple hospital admissions are not uncommon before the diagnosis of WD [11]. According to our previous clinical experience in Whipple's disease [10, 12], our patient's history and the spectrum of neuro-cognitive features (mild ataxic walking, impaired vertical gaze, dysarthria and myoclonic movements, cognitive impairment, depressive mood) before and during his hospitalization, initial clinical and laboratory findings (which ruled out other diagnosis as possible), and proposed diagnostic procedures in CNS Whipple's disease [13], we thought that Whipple's disease should be included as a possible cause of patient's signs and symptoms during the last years and thus laboratory examination for Tropheryma Whipplei [14] was performed that confirmed the diagnosis. An unusual case of Whipple's disease without CNS involvement presented with a broad spectrum of clinical symptoms and prolonged disease duration of approximately 30 years with multiple relapses (e.g. four relapses) has been recently presented [15]. On other hand, isolated CNS Whipple's disease can been diagnosed even in the absence of typical MRI findings $[16,17]$.

We report an unusual case of WD, marked by a broad spectrum of clinical symptoms and prolonged disease duration (nearly 30 years) with 4 successive relapses.

PCR has been emerged as a way to establish the diagnosis of WD in the setting of non-diagnostic tissue biopsies. In our case after the confirmation of possible $\mathrm{CNS}$ WD (fever of unknown origin, chronic migratory arthralgias, unexplained lymphadenopathy, supranuclear vertical gaze palsy, rhythmic myoclonus, severe cognitive deficits and psychiatric manifestations, hypothalamic manifestations), duodenal biopsy and PCR analysis of stools, blood and CSF was conducted. The duodenal biopsy specimen was not remarkable (PAS negative, electron microscopy not performed) but PCR analysis of stools and blood was positive. Thus, the diagnosis of definite WD with CNS involvement was confirmed, according to the diagnostic guidelines proposed by Louis et al. [13].

In order to confirm the diagnosis of possible CNS WD [13], the patient "must have any 1 of 4 systemic symptoms, not due to another known etiology: 1) fever of unknown origin, 2) gastrointestinal symptoms (steatorrhea, chronic diarrhea, abdominal distention, or pain), 3) chronic migratory arthralgias or polyarthralgias and also must have any 1 of 4 neurological signs, not due to another known etiology: 1) supranuclear vertical gaze palsy, rhythmic myoclonus, 3) dementia with psychiatric symptoms, 4) hypothalamic manifestations". For the diagnosis of definite CNS WD [13], the patient "must have any 1 of the following 3 criteria: 1) oculomasticatory myorhythmia or oculo-facialskeletal myorhythmia, 2) positive tissue biopsy, 3) positive PCR analysis. If histological or PCR analysis was not performed on CNS tissue, then the patient must also demonstrate neurological signs. If histological or PCR analysis was performed on CNS tissue, then the patient need not demonstrate neurological signs". Our patient met 7 out of 8 diagnostic criteria for possible WD with CNS involvement [13]: fever of unknown origin, chronic migratory arthralgias, unexplained lymphadenopathy, supranuclear vertical gaze palsy, rhythmic myoclonus, severe cognitive deficits and psychiatric manifestations, hypothalamic manifestations. Positive PCR findings in stools and blood confirmed the diagnosis of definite WD with CNS involvement, considering the presence of neurological signs as well [13].

It has been reported that a couple of factors may contribute to a negative intestine biopsy, namely the absence of chronic diarrhea, lack of endoscopic guidance, specimens not examine by electron microscopy, biopsy not repeated at least once if initially negative [18]. Three of the previous factors are confirmed in our case (absence of chronic diarrhea, electron microscopy not performed, biopsy not repeated after the initial negative findings). Furthermore, Panegyres and colleagues (2006) reviewing the literature and presenting 19 patients with primary WD of the brain without systemic involvement (in contrast to our patient) found three patients with negative or inconclusive PCR on CSF [19]. 
Thus, even in cases with primary CNS WD without any gastrointestinal or other systemic involvement CSF PCR may be negative. On the other hand, it has recently been suggested that a negative PCR result or negative finding on small bowel biopsy do not completely exclude the diagnosis of WD with CNS involvement [20].

Undiagnosed WD may result in multiple admissions to hospitals and secondary extra-gastrointestinal manifestations. In conclusion, our case report might serve as a reminder for clinicians to consider WD in patients with CNS signs, previous episodes of arthralgia, fever and gastrointestinal symptoms and laboratory evidence of active infection after having excluded common differentials.

\section{CONFLICT OF INTEREST}

The authors confirm that this article content has no conflict of interest.

\section{ACKNOWLEDGEMENTS}

Declared none.

\section{REFERENCES}

[1] Dutly F, Altwegg M. Whipple's disease and "Tropheryma whippelii". Clin Microbiol Rev 2001; 14: 561-83.

[2] Ramzan NN, Loftus E Jr, Burgart LJ, et al. Diagnostic and monitoring of Whipple's disease by polymerase chain reaction. Ann Intern Med 1997; 126: 520-7.

[3] Schmitt BP, Richardson H, Smith E, Kaplan R. Encephalopathy complicating Whipple's disease: failure to respond to antibiotics. Ann Intern Med 1981; 94: 51-2.

[4] Panegyres PK, Foster JK, Fallon M, Connor C. The amnesic syndrome of primary Whipple disease of the brain. Cog Behav Neurol 2010; 23: 49-51.

[5] Fleming JL, Wiesner RH, Shorter RG. Whipple's disease: clinical, biochemical, and histopathological features and assessment of treatment in 29 patients. Mayo Clin Proc 1988; 63: 539-51.
[6]

Vital Durand D, Lecomte C, Cathebras P, Rousset H, Godeau P. SNFMI Research Group on Whipple Disease. Whipple disease. Clinical review of 52 cases. Medicine (Baltimore) 1997; 76: 17084.

[7] Gerard A, Sarrot-Reynauld F, Liozon E, et al. Neurologic presentation of Whipple disease: report of 12 cases and review of the literature. Medicine (Baltimore) 2002; 81: 443-57.

[8] Keinath R, Merell DE, Vlietstra RE, Dobbins WO III. Antibiotic treatment and relapse in Whipple's disease: long term follow-up of 88 patients. Gastroenterology 1985; 88: 1867-73.

[9] Brandle M, Ammann P, Spinas GA, et al. Relapsing Whipple's disease presenting with hypopituitarism. Clin Endocrinol 1999; 50: 399-403

[10] Stamboulis E, Kararizou E, Manta P, Grivas I. Segmental myoclonus in Whipple's disease. Electromyogr Clin Neurophysiol 1995; 35: 113-6.

[11] Magira EE, Gounaris T, Sioula E. Whipple's disease: multiple hospital admissions of a man with diarrhoea, fever and arthralgia. J Infect 2005; 51: E35-E37.

[12] Stamboulis E, Manta P, Kararizou E, Grivas I. Whipple's disease with tubular aggregates in asymptomatic muscle. Clin Neuropathol 1993; 12: 121-4.

[13] Louis ED, Lynch T, Kaufmann P, Fahn S, Odel J. Diagnostic guidelines in Central Nervous System Whipple's disease. Ann Neurol 1996; 40: 561-8.

[14] Cohen L, Berther K, Danga C, et al. Polymerase chain reaction of CSF to diagnose Whipple's disease. Lancet 1996; 347: 329

[15] Lochouam M, Lagier JC, Raoult D, Pulcini C. A case of Whipple's disease evolving over 30 years. Med Mal Infect 2014; 44: 331-3.

[16] Compain C, Sacre K, Puechal X, et al. Central nervous system involvement in Whipple disease: clinical study of 18 patients and long-term follow-up. Medicine (Baltimore) 2013; 92: 324-30.

[17] Sung VW, Lyerly MJ, Fallon KB, Bashir K. Isolated CNS Whipple disease with normal brain MRI and false-positive CSF 14-3-3 protein: a case report and review of the literature. Brain Behav 2012; 2: 838-43.

[18] Louis ED. Whipple Disease. In: Rowland LP, Pedley TA, Eds. Merritt's Neurology. Lippincott Williams \& Wilkins 2010; pp. 248-9

[19] Panegyres PK, Edis E, Beamn M, Fallon M. Primary Whipple's disease of the brain: characterization of the clinical syndrome and molecular diagnosis. QJM 2006; 99: 609-23.

[20] Black DF, Aksamitb AJ, Morrisa JM. MR imaging of Central Nervous System Whipple disease: a 15-Year review. AJNR 2010; 31: 1493-7.

(C) Zalonis et al.; Licensee Bentham Open.

This is an open access article licensed under the terms of the Creative Commons Attribution Non-Commercial License (http://creativecommons.org/licenses/bync/3.0/), which permits unrestricted, non-commercial use, distribution and reproduction in any medium, provided the work is properly cited. 